\title{
RARE DECAYS AND CP VIOLATION BEYOND THE STANDARD MODEL
}

\author{
LUCA SILVESTRINI \\ INFN, Sez. di Roma, Dip. di Fisica, Univ. di Roma "La Sapienza", \\ P.le A. Moro, I-00185 Rome, Italy.E-mail: Luca.Silvestrini@roma1.infn.it
}

\begin{abstract}
We review the status of rare decays and CP violation in extensions of the Standard Model. We analyze the determination of the unitarity triangle and the model-independent constraints on new physics that can be derived from this analysis. We find stringent bounds on new contributions to $K-\bar{K}$ and $B_{d}-\bar{B}_{d}$ mixing, pointing either to models of minimal flavour violation or to models with new sources of flavour and CP violation in $b \rightarrow s$ transitions. We discuss the status of the universal unitarity triangle in minimal flavour violation, and study rare decays in this class of models. We then turn to supersymmetric models with nontrivial mixing between second and third generation squarks, discuss the present constraints on this mixing and analyze the possible effects on CP violation in $b \rightarrow s$ nonleptonic decays and on $B_{s}-\bar{B}_{s}$ mixing. We conclude presenting an outlook on Lepton-Photon 2009 .
\end{abstract}

\section{Introduction}

The Standard Model (SM) of electroweak and strong interactions works beautifully up to the highest energies presently explored at colliders. However, there are several indications that it must be embedded as an effective theory into a more complete model that should, among other things, contain gravity, allow for gauge coupling unification and provide a dark matter candidate and an efficient mechanism for baryogenesis. This effective theory can be described by the Lagrangian

$\mathcal{L}\left(M_{W}\right)=\Lambda^{2} H^{\dagger} H+\mathcal{L}_{\mathrm{SM}}+\frac{1}{\Lambda} \mathcal{L}^{5}+\frac{1}{\Lambda^{2}} \mathcal{L}^{6}+\ldots$, where the logarithmic dependence on the cutoff $\Lambda$ has been neglected. Barring the possibility of a conspiracy between physics at scales below and above $\Lambda$ to give an electroweak symmetry breaking scale $M_{W} \ll \Lambda$, we assume that the cutoff lies close to $M_{W}$. Then the power suppression of higher dimensional operators is not too severe for $\mathcal{L}^{5,6}$ to produce sizable effects in low-energy processes, provided that they do not compete with tree-level SM contributions. Therefore, we should look for new physics effects in quantities that in the SM are zero at the tree level and are finite and calculable at the quantum level. Within the SM, such quantities fall in two categories: i) electroweak precision observables (protected by the electroweak symmetry) and ii) Flavour Changing Neutral Currents (FCNC) (protected by the GIM mechanism). The first category has been discussed by S. Dawson at this conference, while the second will be analyzed here.

In the SM, all FCNC and $\mathrm{CP}$ violating processes are computable in terms of quark masses and of the elements of the CabibboKobayashi-Maskawa (CKM) matrix. This implies very strong correlations among observables in the flavour sector. New Physics (NP) contributions, or equivalently the operators in $\mathcal{L}^{5,6}$, violate in general these correlations, so that NP can be strongly constrained by combining all the available experimental information on flavour and $\mathrm{CP}$ violation.

\section{The UT analysis beyond the SM}

A very useful tool to combine the available experimental data in the quark sector is the Unitarity Triangle (UT) analysis. ${ }^{1,2}$ Thanks to the measurements of the UT angles recently performed at $B$ factories, which provide a determination of the UT comparable in accuracy with the one performed using the other available data, the UT fit is now overconstrained (see Fig. 1). It is therefore become possible to add NP contributions to all quantities entering the UT analysis and to 

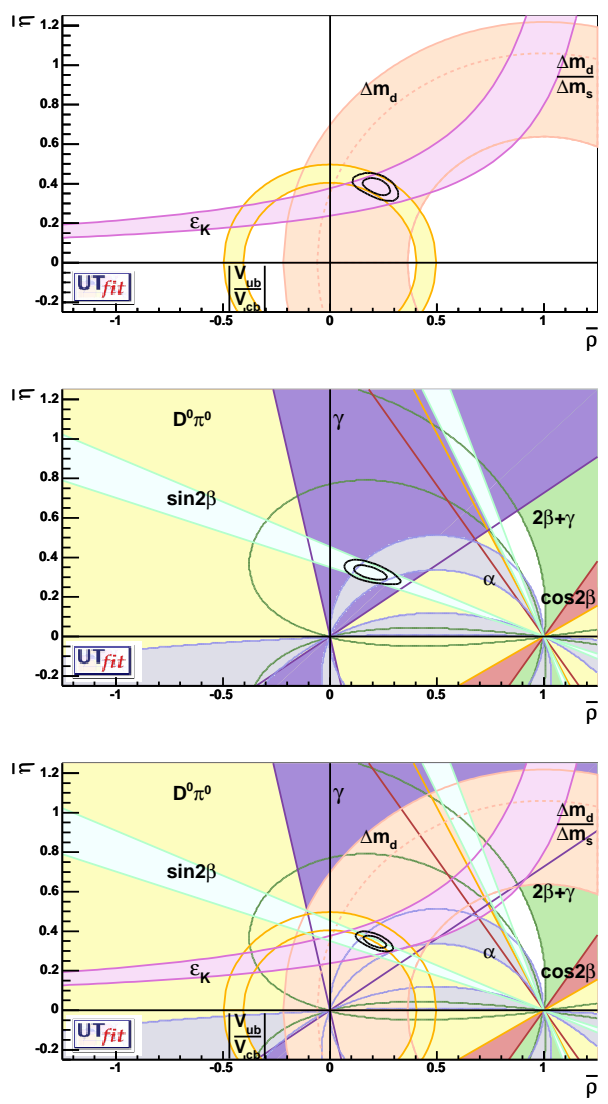

Figure 1. The UT obtained without using (top) and using only (center) the measurements of the UT angles, and the combined fit result (bottom).

perform a combined fit of NP contributions and SM parameters. In general, NP models introduce a large number of new parameters: flavour changing couplings, short distance coefficients and matrix elements of new local operators. The specific list and the actual values of these parameters can only be determined within a given model. Nevertheless, each of the meson-antimeson mixing processes is described by a single amplitude and can be parameterized, without loss of generality, in terms of two parameters, which quantify the difference between the full amplitude and the SM one. ${ }^{3}$ Thus, for instance, in the case of $B_{q}^{0}-\bar{B}_{q}^{0}$ mixing we define

$$
C_{B_{q}} e^{2 i \phi_{B_{q}}}=\frac{\left\langle B_{q}^{0}\left|H_{\mathrm{eff}}^{\mathrm{full}}\right| \bar{B}_{q}^{0}\right\rangle}{\left\langle B_{q}^{0}\left|H_{\mathrm{eff}}^{\mathrm{SM}}\right| \bar{B}_{q}^{0}\right\rangle},(q=d, s)
$$

where $H_{\mathrm{eff}}^{\mathrm{SM}}$ includes only the SM box dia-

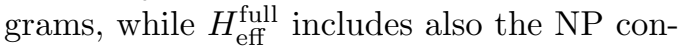
tributions. As far as the $K^{0}-\bar{K}^{0}$ mixing is concerned, we find it convenient to introduce a single parameter which relates the imaginary part of the amplitude to the SM one:

$$
C_{\epsilon_{K}}=\frac{\operatorname{Im}\left[\left\langle K^{0}\left|H_{\mathrm{eff}}^{\mathrm{full}}\right| \bar{K}^{0}\right\rangle\right]}{\operatorname{Im}\left[\left\langle K^{0}\left|H_{\mathrm{eff}}^{\mathrm{SM}}\right| \bar{K}^{0}\right\rangle\right]} .
$$

Therefore, all NP effects in $\Delta F=2$ transitions are parameterized in terms of three real quantities, $C_{B_{d}}, \phi_{B_{d}}$ and $C_{\epsilon_{K}}$. NP in the $B_{s}$ sector is not considered, due to the lack of experimental information, since both $\Delta m_{s}$ and $A_{\mathrm{CP}}\left(B_{s} \rightarrow J / \psi \phi\right)$ are not yet measured.

$\mathrm{NP}$ effects in $\Delta B=1$ transitions can also affect some of the measurements entering the UT analysis, in particular the measurements of $\alpha$ and $A_{\mathrm{SL}} \cdot{ }^{4}$ However, under the hypothesis that NP contributions are mainly $\Delta I=1 / 2$, their effect can be taken into account in the fit of the $B \rightarrow \pi \pi, \rho \pi, \rho \rho$ decay amplitudes. Concerning $A_{\mathrm{SL}}$, penguins only enter at the Next-to-Leading order and therefore NP in $\Delta B=1$ transitions produces subdominant effects with respect to the leading $\Delta B=2$ contribution.

The results obtained in a global fit for $C_{B_{d}}, C_{\epsilon_{K}}, C_{B_{d}}$ vs. $\phi_{B_{d}}$, and $\gamma$ vs. $\phi_{B_{d}}$ are shown in Fig. 2, together with the corresponding regions in the $\bar{\rho}-\bar{\eta}$ plane. ${ }^{4}$

To illustrate the impact of the various constraints on the analysis, in Fig. 3 we show the selected regions in the $\phi_{B_{d}}$ vs. $C_{B_{d}}$ and $\phi_{B_{d}}$ vs. $\gamma$ planes using different combinations of constraints. The first row represents the pre-2004 situation, when only $\left|V_{u b} / V_{c b}\right|$, $\Delta m_{d}, \varepsilon_{K}$ and $\sin 2 \beta$ were available, selecting a continuous band for $\phi_{B_{d}}$ as a function of $\gamma$ and a broad region for $C_{B_{d}}$. Adding the determination of $\gamma$ (second row), only four regions in the $\phi_{B_{d}}$ vs. $\gamma$ plane survive, 

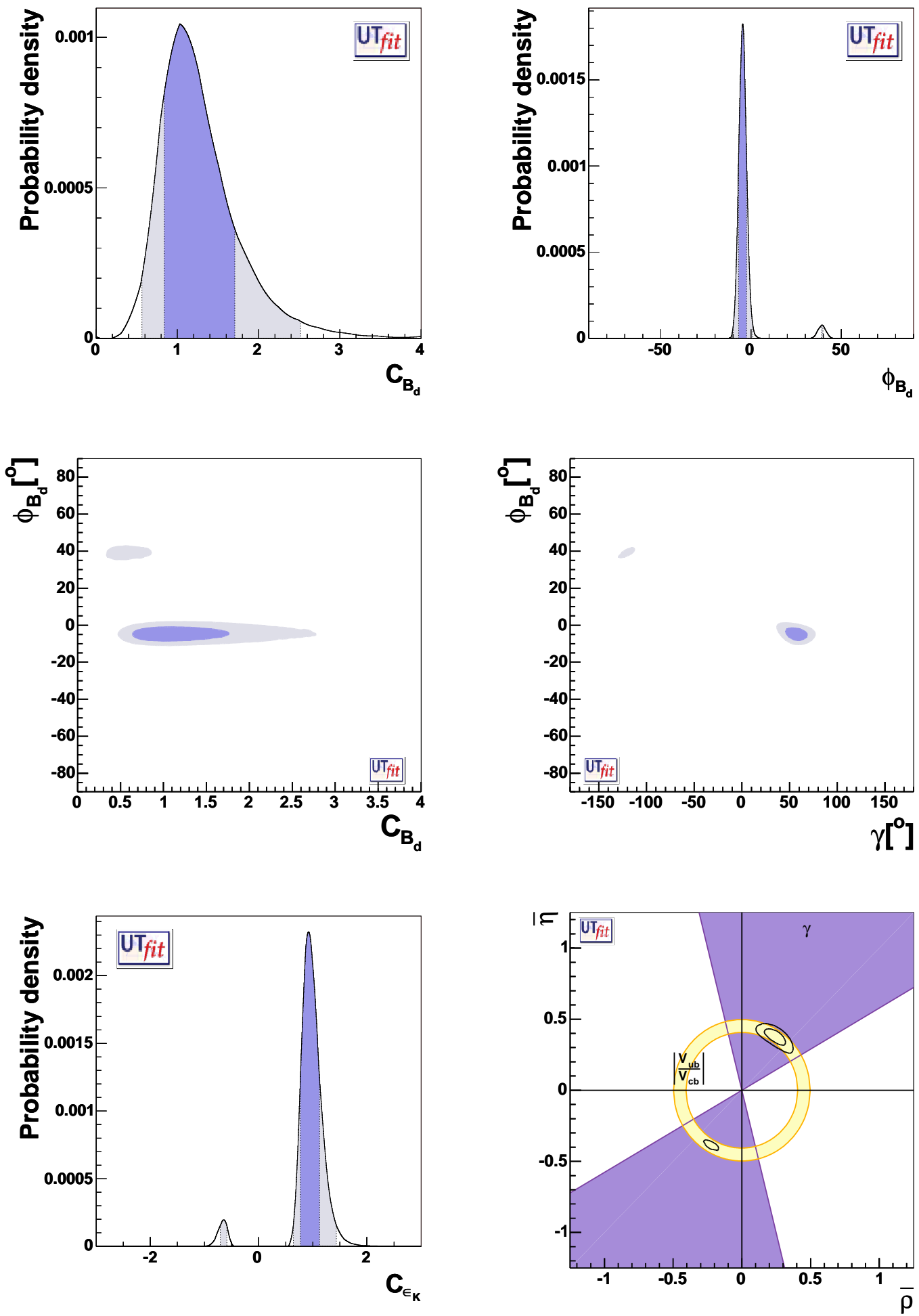

Figure 2. From top to bottom and from left to right, p.d.f.'s for $C_{B_{d}}, \phi_{B_{d}}, \phi_{B_{d}} v s . C_{B_{d}}, \phi_{B_{d}} v s . \gamma, C_{\epsilon_{K}}$ and the selected region on the $\bar{\rho}-\bar{\eta}$ plane obtained from the NP analysis. In the last plot, selected regions corresponding to $68 \%$ and $95 \%$ probability are shown, together with $95 \%$ probability regions for $\gamma$ (from $D K$ final states) and $\left|V_{u b} / V_{c b}\right|$. Dark (light) areas correspond to the $68 \%(95 \%)$ probability region. 

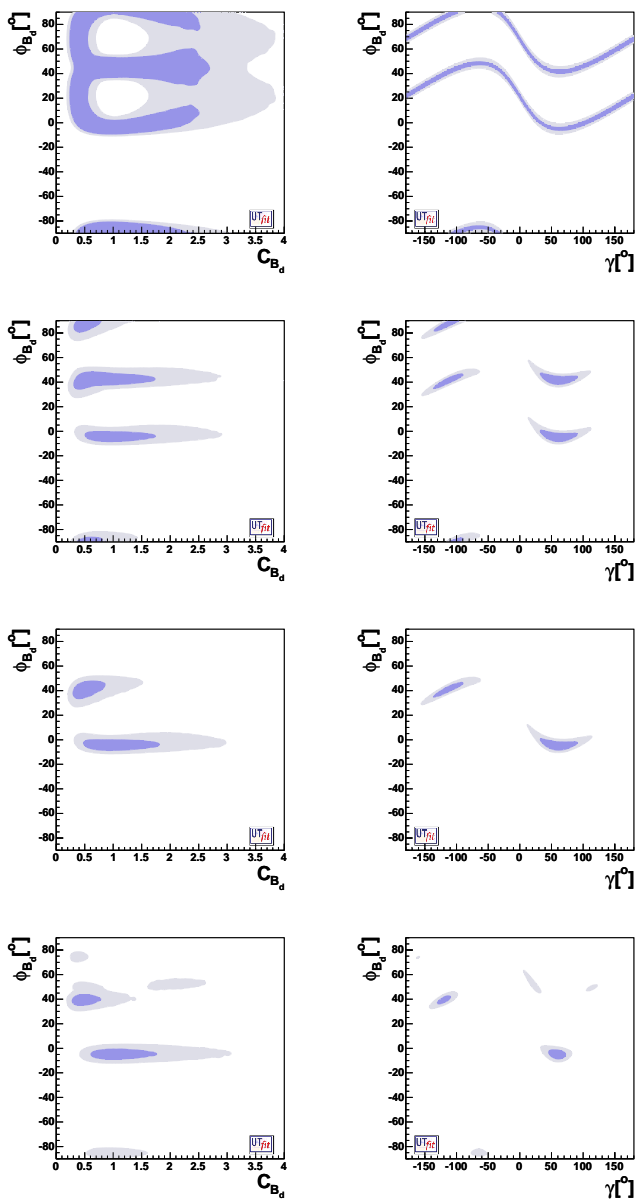

Figure 3. From top to bottom: distributions of $\phi_{B_{d}}$ vs. $C_{B_{d}}$ (left) and $\phi_{B_{d}}$ vs. $\gamma$ (right) using the following constraints: i) $\left|V_{u b} / V_{c b}\right|, \Delta m_{d}, \varepsilon_{K}$ and $\sin 2 \beta$; ii) the constraints in i) plus $\gamma$; iii) the constraints in ii) plus $\cos 2 \beta$ from $B_{d} \rightarrow J / \psi K^{*}$ and $\beta$ from $B \rightarrow D h^{0}$; iv) the constraints in ii) plus $\alpha$.

two of which overlap in the $\phi_{B_{d}}$ vs. $C_{B_{d}}$ plane. Two of these solutions have values of $\cos 2\left(\beta+\phi_{B_{d}}\right)$ and $\alpha-\phi_{B_{d}}$ different from the SM predictions, and are therefore disfavoured by $(\cos 2 \beta)^{\exp }$ and by the measurement of $(2 \beta)^{\exp }$ from $B \rightarrow D h^{0}$ decays, and by $\alpha^{\exp }$ (third and fourth row respectively). On the other hand, the remaining solution has a very large value for $A_{\mathrm{SL}}$ and is therefore disfavoured by $A_{\mathrm{SL}}^{\mathrm{exp}}$, leading to the final results already presented in Fig. 2. The numerical results of the analysis can be found

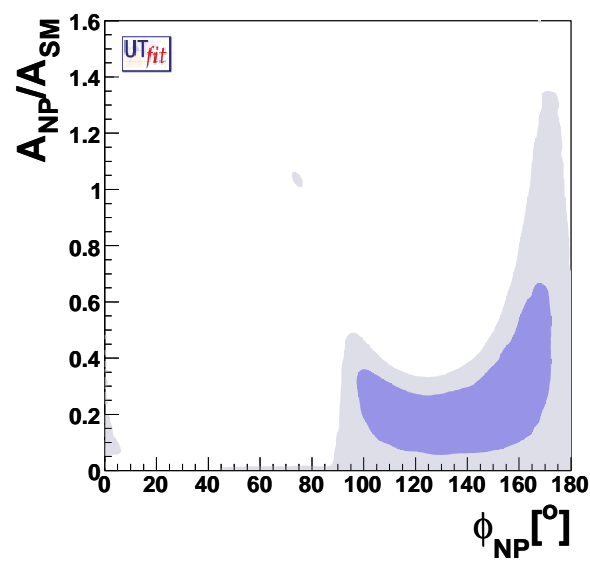

Figure 4. P.d.f. in the $\left(A_{\mathrm{NP}} / A_{\mathrm{SM}}\right)$ vs. $\phi_{\mathrm{NP}}$ plane for NP in the $|\Delta B|=2$ sector (see Eq. (3)).

in ref. ${ }^{4}$ (see ref. ${ }^{2,5}$ for previous analyses).

Before concluding this section, let us analyze more in detail the results in Fig. 2. Writing

$$
C_{B_{d}} e^{2 i \phi_{B_{d}}}=\frac{A_{\mathrm{SM}} e^{2 i \beta}+A_{\mathrm{NP}} e^{2 i\left(\beta+\phi_{\mathrm{NP}}\right)}}{A_{\mathrm{SM}} e^{2 i \beta}},
$$

and given the p.d.f. for $C_{B_{d}}$ and $\phi_{B_{d}}$, we can derive the p.d.f. in the $\left(A_{\mathrm{NP}} / A_{\mathrm{SM}}\right)$ vs. $\phi_{\mathrm{NP}}$ plane. The result is reported in Fig. 4. We see that the NP contribution can be substantial if its phase is close to the SM phase, while for arbitrary phases its magnitude has to be much smaller than the SM one. Notice that, with the latest data, the $\mathrm{SM}\left(\phi_{B_{d}}=0\right)$ is disfavoured at $68 \%$ probability due to a slight disagreement between $\sin 2 \beta$ and $\left|V_{u b} / V_{c b}\right|$. This requires $A_{\mathrm{NP}} \neq 0$ and $\phi_{\mathrm{NP}} \neq 0$. For the same reason, $\phi_{\mathrm{NP}}>90^{\circ}$ at $68 \%$ probability and the plot is not symmetric around $\phi_{\mathrm{NP}}=90^{\circ}$.

Assuming that the small but nonvanishing value for $\phi_{B_{d}}$ we obtained is just due to a statistical fluctuation, the result of our analysis points either towards models with no new source of flavour and $\mathrm{CP}$ violation beyond the ones present in the SM (Minimal Flavour Violation, MFV), or towards 
models in which new sources of flavour and CP violation are only present in $b \rightarrow s$ transitions. In the rest of this talk we will consider these two possibilities, starting from the former.

\section{MFV models}

We now specialize to the case of MFV. Making the basic assumption that the only source of flavour and CP violation is in the Yukawa couplings, ${ }^{6}$ it can be shown that the phase of $|\Delta B|=2$ amplitudes is unaffected by NP, and so is the ratio $\Delta m_{s} / \Delta m_{d}$. This allows the determination of the Universal Unitarity Triangle independent on NP effects, based on $\left|V_{u b} / V_{c b}\right|, \gamma, A_{C P}\left(B \rightarrow J / \Psi K^{(*)}\right), \beta$ from $B \rightarrow D^{0} h^{0}, \alpha$, and $\Delta m_{s} / \Delta m_{d}{ }^{7}$ We present here the determination of the UUT, which is independent of NP contributions in the context of MFV models. The details of the analysis and the upper bounds on NP contributions that can be derived from it can be found in ref. ${ }^{4}$

In Fig. 5 we show the allowed region in the $\bar{\rho}-\bar{\eta}$ plane for the UUT. The corresponding values and ranges are reported in Tab. 1. The most important differences with respect to the general case are that i) the lower bound on $\Delta m_{s}$ forbids the solution in the third quadrant, and ii) the constraint from $\sin 2 \beta$ is now effective, so that we are left with a region very similar to the SM one.

Table 1. Results of the UUT analysis.

\begin{tabular}{ccc}
\hline & UUT (68\%) & UUT $(95 \%)$ \\
\hline $\bar{\rho}$ & $0.259 \pm 0.068$ & {$[0.107,0.376]$} \\
$\bar{\eta}$ & $0.320 \pm 0.042$ & {$[0.241,0.399]$} \\
$\sin 2 \beta$ & $0.728 \pm 0.031$ & {$[0.668,0.778]$} \\
$\alpha\left[^{\circ}\right]$ & $105 \pm 11$ & {$[81,124]$} \\
$\gamma\left[^{\circ}\right]$ & $51 \pm 10$ & {$[33,75]$} \\
{$[2 \beta+\gamma]\left[^{\circ}\right]$} & $98 \pm 12$ & {$[77,123]$} \\
$\Delta m_{s}\left[\mathrm{ps}^{-1}\right]$ & $20.6 \pm 5.6$ & {$[10.6,32.6]$} \\
\hline
\end{tabular}

Starting from the determination of the UUT, one can study rare decays in MFV

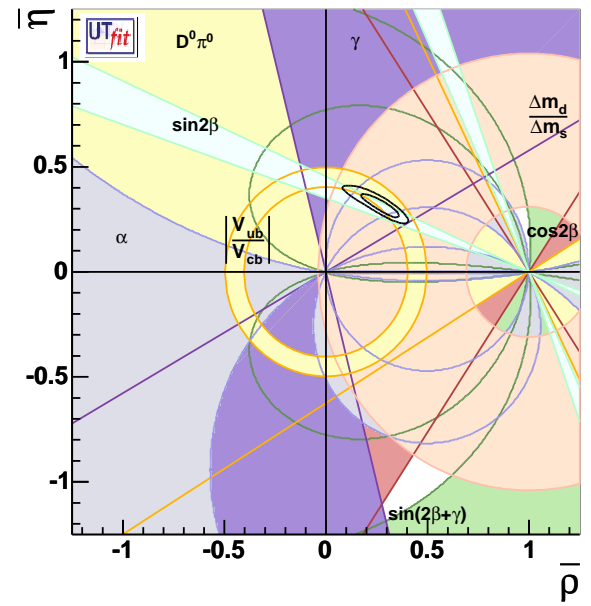

Figure 5. The selected region on $\bar{\rho}-\bar{\eta}$ plane obtained from the determination of the UUT.

models. ${ }^{8}$ In general, a model-independent analysis of rare decays is complicated by the large number of higher dimensional operators that can contribute beyond the SM. ${ }^{9}$ The situation drastically simplifies in MFV models, where (excluding large $\tan \beta$ scenarios) no new operators arise beyond those generated by $W$ exchange. Since the mass scale of NP must be higher than $M_{W}$, we can further restrict our attention to operators up to dimension five, since higher dimensional operators will suffer a stronger suppression by the scale of NP. In this way, we are left with NP contributions to two operators only: the FCNC $Z$ and magnetic vertices. ${ }^{a} \mathrm{NP}$ contributions can be reabsorbed in a redefinition of the SM coefficients of these operators: $C=C_{\mathrm{SM}}+\Delta C$ for the $Z$ vertex and $C_{7}^{\text {eff }}=C_{7 \mathrm{SM}}^{\mathrm{eff}}+\Delta C_{7}^{\mathrm{eff}}$ for the magnetic operator. $^{b}$

The analysis goes as follows: using

\footnotetext{
$\bar{a}$ The chromomagnetic vertex should also be considered, but this is not necessary for the analysis presented here. ${ }^{8}$

${ }^{b}$ We find it convenient to redefine the $C$ function at the electroweak scale, and the $C_{7}^{\text {eff }}$ function at the hadronic scale.
} 
the CKM parameters as determined by the UUT analysis, one can use $\operatorname{BR}\left(B \rightarrow X_{s} \gamma\right)$, $\operatorname{BR}\left(B \rightarrow X_{s} l^{+} l^{-}\right)$and $\operatorname{BR}\left(K^{+} \rightarrow \pi^{+} \nu \bar{\nu}\right)$ to constrain $\Delta C$ and $\Delta C_{7}^{\text {eff }}$. Then, predictions can be obtained for all other $K$ and $B$ rare decays. Fig. 6 shows the constraints on the NP contributions. Three possibilities emerge: i) the SM-like solution with NP corrections close to zero; ii) the "opposite $C$ " solution with the sign of $C$ flipped by NP and $C_{7}^{\text {eff }}$ close to the SM value; iii) the "opposite $C_{7}$ " solution with the sign of $C_{7}^{\text {eff }}$ flipped, which however requires a sizable deviation from the SM also in $C$.

The corresponding predictions for other rare decays are reported in Fig. 7, and the $95 \%$ probability upper bounds are summarized in Tab. 2, together with the SM predictions obtained starting from the UUT analysis. It is clear that, given present constraints, rare decays can be only marginally enhanced with respect to the SM, while strong suppressions are still possible. Future improvements in the measurements of $\operatorname{BR}\left(B \rightarrow X_{s} \gamma\right)$, $\mathrm{BR}\left(B \rightarrow X_{s} l^{+} l^{-}\right)$and $\mathrm{BR}\left(K^{+} \rightarrow \pi^{+} \nu \bar{\nu}\right)$ will help us to reduce the allowed region for NP contributions. Another very interesting observable is the Forward-Backward asymmetry in $B \rightarrow X_{s} l^{+} l^{-} .{ }^{10}$ Indeed, the two solutions for $\Delta C_{7}^{\text {eff }}$ and the corresponding possible values of $\Delta C$ give rise to different profiles of the normalized $\bar{A}_{\mathrm{FB}}$ (see eq. (3.10) of ref. ${ }^{8}$, where more details can be found). This can be seen explicitly in Fig. 8.

\section{New Physics in $\mathrm{b} \rightarrow \mathrm{s}$ transitions}

We concluded sec. 2 pointing out two possible NP scenarios favoured by the UT analysis: the first one, MFV, was discussed in the previous section, now we turn to the second one, i.e. models with new sources of flavour and $\mathrm{CP}$ violation in $b \rightarrow s$ transitions. Indeed, most NP models fall in this class. Since the SM flavour $S U(3)$ symmetry is strongly broken by the top (and bottom) Yukawa cou-
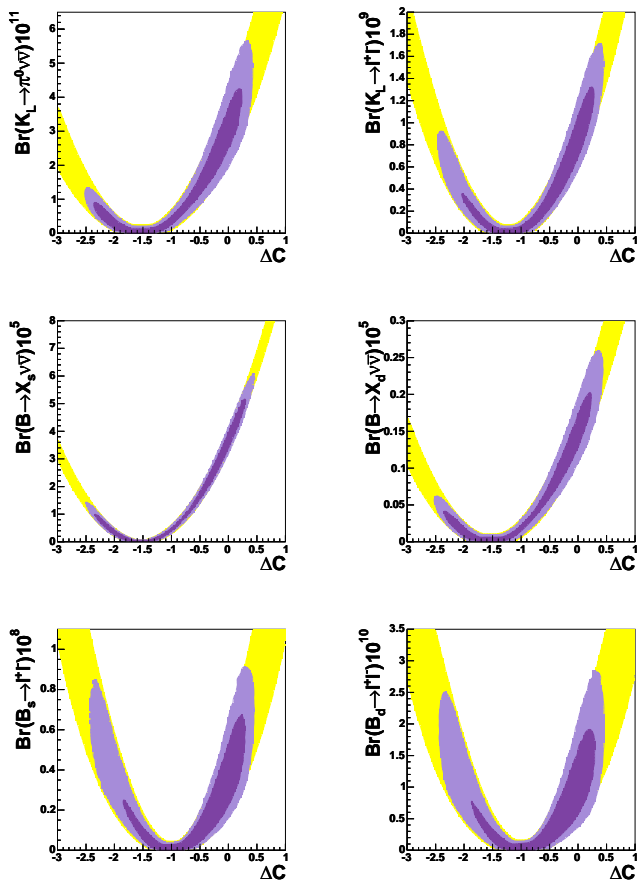

Figure 7. P.d.f.'s for the branching ratios of the rare decays $\operatorname{Br}\left(K_{L} \rightarrow \pi^{0} \nu \bar{\nu}\right), \operatorname{Br}\left(K_{L} \rightarrow \mu \bar{\mu}\right)_{\mathrm{SD}}, \operatorname{Br}(B \rightarrow$ $\left.X_{d, s} \nu \bar{\nu}\right)$, and $\operatorname{Br}\left(B_{d, s} \rightarrow \mu^{+} \mu^{-}\right)$as a function of $\Delta C$. Dark (light) areas correspond to the $68 \%(95 \%)$ probability region. Very light areas correspond to the range obtained without using the experimental information.

plings, flavour models are not very effective in constraining NP contributions to $b \rightarrow s$ transitions. ${ }^{12}$ The same happens in models of gauge-Higgs unification or composite Higgs models, due to the large coupling between the third generation and the EW symmetry breaking sector. ${ }^{13}$ Last but not least, the large atmospheric neutrino mixing angle suggests the possibility of large NP contributions to $b \rightarrow s$ processes in SUSY-GUTs. ${ }^{14}$

This well-motivated scenario is becoming more and more interesting since $B$ factories are probing NP effects in $b \rightarrow s$ penguin transitions, and the Tevatron and $\mathrm{LHCb}$ will probe $\mathrm{NP}$ effects in $B_{s}-\bar{B}_{s}$ mixing in the near future. For the latter process, there is a solid SM prediction which states that $\Delta m_{s}>28(30) \mathrm{ps}^{-1}$ implies NP at $2 \sigma(3 \sigma)$. 

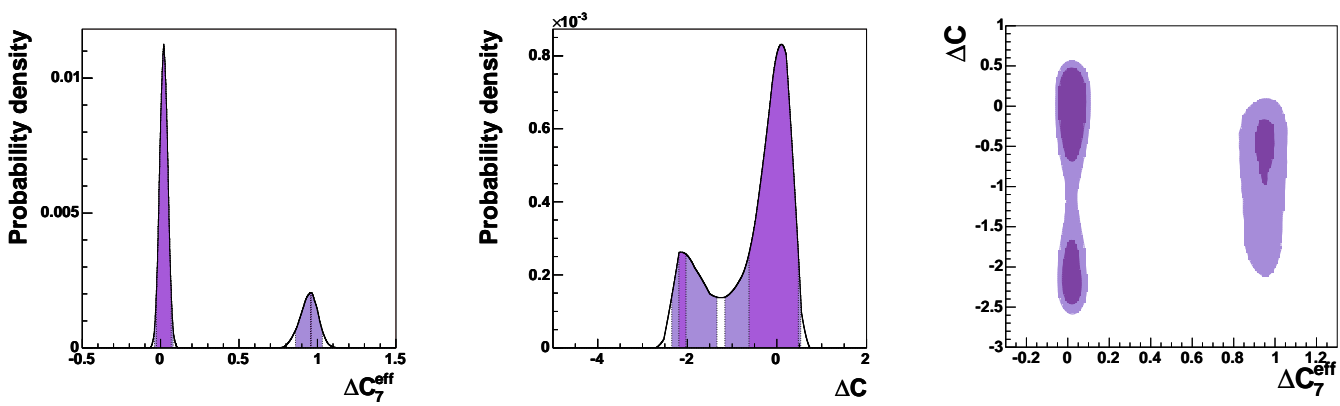

Figure 6. P.d.f.'s for $\Delta C_{7}^{\text {eff }}$ (left), $\Delta C$ (middle) and $\Delta C$ vs. $\Delta C_{7}^{\text {eff }}$ (right).

Table 2. Upper bounds for rare decays in MFV models at $95 \%$ probability, the corresponding values in the SM (using inputs from the UUT analysis) and the available experimental information.

\begin{tabular}{|c|c|c|c|c|}
\hline Branching Ratios & MFV $(95 \%)$ & SM (68\%) & SM (95\%) & $\exp ^{11}$ \\
\hline$B r\left(K^{+} \rightarrow \pi^{+} \nu \bar{\nu}\right) \times 10^{11}$ & $<11.9$ & $8.3 \pm 1.2$ & $(6.1,10.9)$ & $\left(14.7_{-8.9}^{+13.0}\right)$ \\
\hline$B r\left(K_{L} \rightarrow \pi^{0} \nu \bar{\nu}\right) \times 10^{11}$ & $<4.59$ & $3.08 \pm 0.56$ & $(2.03,4.26)$ & $<5.9 \cdot 10^{4}$ \\
\hline$B r\left(K_{L} \rightarrow \mu \bar{\mu}\right)_{\mathrm{SD}} \times 10^{9}$ & $<1.36$ & $0.87 \pm 0.13$ & $(0.63,1.15)$ & - \\
\hline$B r\left(B \rightarrow X_{s} \nu \bar{\nu}\right) \times 10^{5}$ & $<5.17$ & $3.66 \pm 0.21$ & $(3.25,4.09)$ & $<64$ \\
\hline$B r\left(B \rightarrow X_{d} \nu \bar{\nu}\right) \times 10^{6}$ & $<2.17$ & $1.50 \pm 0.19$ & $(1.12,1.91)$ & $<2.2 \cdot 10^{2}$ \\
\hline$B r\left(B_{s} \rightarrow \mu^{+} \mu^{-}\right) \times 10^{9}$ & $<7.42$ & $3.67 \pm 1.01$ & $(1.91,5.91)$ & $<1.5 \cdot 10^{2}$ \\
\hline$B r\left(B_{d} \rightarrow \mu^{+} \mu^{-}\right) \times 10^{10}$ & $<2.20$ & $1.04 \pm 0.34$ & $(0.47,1.81)$ & $<3.9 \cdot 10^{2}$ \\
\hline
\end{tabular}
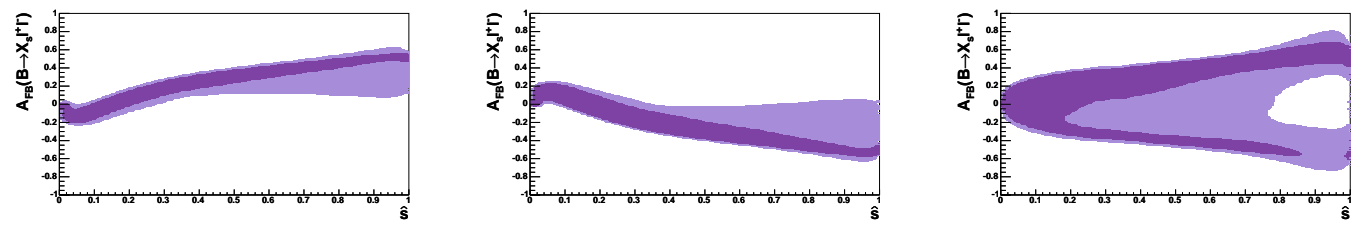

Figure 8. P.d.f. for the normalized forward-backward asymmetry in $B \rightarrow X_{s} l^{+} l^{-}$for $\Delta C_{7}^{\text {eff }} \sim 0$ with $\Delta C>-1$ (left), for $\Delta C_{7}^{\text {eff }} \sim 0$ with $\Delta C<-1$ (middle) and for $\Delta C_{7}^{\text {eff }} \sim 1$ (right). Dark (light) areas correspond to the $68 \%$ (95\%) probability region.

For $b \rightarrow s$ penguin transitions, $B \rightarrow X_{s} \gamma$ and $B \rightarrow X_{s} l^{+} l^{-}$decays strongly constrain the FCNC $Z$ and magnetic effective vertices, as already discussed in the previous section in the simplified case of MFV. On the other hand, NP contributions to the chromomagnetic $b \rightarrow s$ vertex and to dimension six operators are only mildly constrained by radiative and semileptonic decays, so that they can contribute substantially to $b \rightarrow s$ hadronic decays, although in any given model all these NP contributions are in general correlated and thus more constrained.

As shown in the talk by $\mathrm{K}$. Abe at this conference, $B$-factories are now probing NP in $b \rightarrow s$ transitions by measuring the coefficient $\mathcal{S}$ of the $\sin \Delta m_{d} t$ term in timedependent $\mathrm{CP}$ asymmetries for $b \rightarrow s$ nonleptonic decays. Neglecting the doubly Cabibbo suppressed $b \rightarrow u$ contributions, one should have $\mathcal{S}=\sin 2 \beta$ for all $b \rightarrow s$ channels within the SM, so that deviations from this equality would signal NP in the decay amplitude. ${ }^{15}$ However, $b \rightarrow u$ terms may also cause devi- 
ations $\Delta \mathcal{S}$ from the equality above, so that the estimate of $\Delta \mathcal{S}$ becomes of crucial importance in looking for NP. While a detailed analysis of $\Delta \mathcal{S}$ goes beyond the scope of this talk, ${ }^{16}$ the reader should be warned that $\Delta \mathcal{S}$ might be quite large for channels that are not pure penguins, and in particular for final states containing $\eta^{\prime}$ mesons. ${ }^{c}$ In this respect, it is of fundamental importance to improve the measurement of pure penguin channels, such as $\phi K_{S}$, as well as to enlarge the sample of available $b \rightarrow s$ and $b \rightarrow d$ channels, in order to be able to use flavour symmetries to constrain $\Delta S$.

The problem of computing $\Delta \mathcal{S}$ in any given NP model is even tougher: as is well known, in the presence of two contributions to the amplitude with different weak phases, $\mathrm{CP}$ asymmetries depend on hadronic matrix elements, which at present cannot be computed in a model-independent way. One has then to resort to models of hadronic dynamics to estimate $\Delta \mathcal{S}$, with the large theoretical uncertainties associated to this procedure.

With the above caveat in mind, let us now focus on SUSY and discuss the phenomenological effects of the new sources of flavour and $\mathrm{CP}$ violation in $b \rightarrow s$ processes that arise in the squark sector. ${ }^{18}$ In general, in the MSSM squark masses are neither flavour-universal, nor are they aligned to quark masses, so that they are not flavour diagonal in the super-CKM basis, in which quark masses are diagonal and all neutral current (SUSY) vertices are flavour diagonal. The ratios of off-diagonal squark mass terms to the average squark mass define four new sources of flavour violation in the $b \rightarrow s$ sector: the mass insertions $\left(\delta_{23}^{d}\right)_{A B}$, with $A, B=L, R$ referring to the helicity of the corresponding quarks. These $\delta$ 's are in general complex, so that they also violate $\mathrm{CP}$.

\footnotetext{
${ }^{c}$ Theoretical uncertainties might be larger than what expected even in the golden mode $B \rightarrow J / \psi K_{S}$, although they can be reduced with the aid of other decay modes. ${ }^{17}$
}

One can think of them as additional CKMtype mixings arising from the SUSY sector. Assuming that the dominant SUSY contribution comes from the strong interaction sector, i.e. from gluino exchange, all FCNC processes can be computed in terms of the SM parameters plus the four $\delta$ 's plus the relevant SUSY masses: the gluino mass $m_{\tilde{g}}$, the average squark mass $m_{\tilde{q}}$ and, in general, $\tan \beta$ and the $\mu$ parameter. ${ }^{d}$ Barring accidental cancellations, one can consider one single $\delta$ parameter, fix the SUSY masses and study the phenomenology. The constraints on $\delta$ 's come at present from BR's and CP asymmetries in $B \rightarrow X_{s} \gamma, B \rightarrow X_{s} l^{+} l^{-}$and from the lower bound on $\Delta m_{s}$. Since gluino exchange does not generate a sizable $\Delta C$ in the notation of the previous section, the combined constraints from radiative and semileptonic decays are particularly stringent.
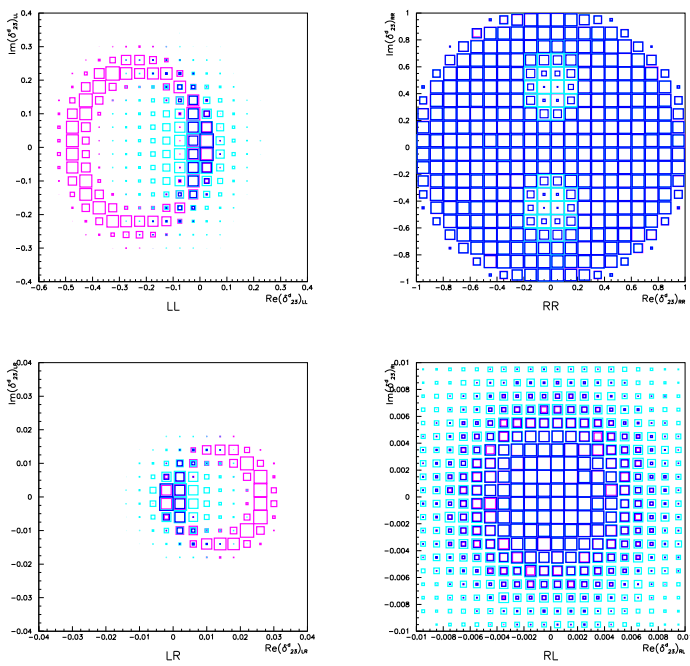

Figure 9. P.d.f.'s in the $\operatorname{Re}\left(\delta_{23}^{d}\right)_{A B}-\operatorname{Im}\left(\delta_{23}^{d}\right)_{A B}$ plane for $A, B=L, R$, as determined by $B \rightarrow X_{s} \gamma$ (violet), $B \rightarrow X_{s} l^{+} l^{-}$(light blue) and all constraints (dark blue).

Fixing as an example $m_{\tilde{g}}=m_{\tilde{q}}=-\mu=$ $350 \mathrm{GeV}$ and $\tan \beta=10$, one obtains the

\footnotetext{
${ }^{d}$ The last two parameters are irrelevant as long as $\tan \beta$ is of $\mathcal{O}(1)$.
} 
constraints on $\delta$ 's reported in Fig. 9. Several comments are in order at this point: i) only $\left(\delta_{23}^{d}\right)_{\text {LL,LR generate amplitudes that }}$ interfere with the SM one in rare decays. Therefore, the constraints from rare decays for $\left(\delta_{23}^{d}\right)_{\mathrm{RL}, \mathrm{RR}}$ are symmetric around zero, while the interference with the SM produces the circular shape of the $B \rightarrow X_{s} \gamma$ constraint on $\left(\delta_{23}^{d}\right)_{\mathrm{LL}, \mathrm{LR}}$. ii) We recall that $\mathrm{LR}$ and $\mathrm{RL}$ mass insertions generate much larger contributions to the (chromo)magnetic operators, since the necessary chirality flip can be performed on the gluino line $\left(\propto m_{\tilde{g}}\right)$ rather than on the quark line $\left(\propto m_{\tilde{b}}\right)$. Therefore, the $B \rightarrow X_{s} \gamma$ constraint is much more effective on these insertions. iii) The $\mu \tan \beta$ flavourconserving LR squark mass term generates, together with a flavour changing LL mass insertion, an effective $\left(\delta_{23}^{d}\right)_{\mathrm{LR}}^{\mathrm{eff}}$ that contributes to $B \rightarrow X_{s} \gamma$. Having chosen a negative $\mu$, we have $\left(\delta_{23}^{d}\right)_{\mathrm{LR}}^{\mathrm{eff}} \propto-\left(\delta_{23}^{d}\right)_{\mathrm{LL}}$ and therefore the circle determined by $B \rightarrow X_{s} \gamma$ in the LL and LR cases lies on opposite sides of the origin (see Fig. 9). iv) For LL and LR cases, $B \rightarrow X_{s} \gamma$ and $B \rightarrow X_{s} l^{+} l^{-}$produce bounds with different shapes on the Re $\delta-\operatorname{Im} \delta$ plane (violet and light blue regions in Fig. 9), so that applying them simultaneously only a much smaller region around the origin survives (dark blue regions in Fig. 9). This shows the key role played by rare decays in constraining new sources of flavour and $\mathrm{CP}$ violation in the squark sector. v) For the RR case, the constraints from rare decays are very weak, so that almost all $\delta$ 's with $\left|\left(\delta_{23}^{d}\right)_{\mathrm{RR}}\right|<1$ are allowed, except for two small forbidden regions where $\Delta m_{s}$ goes below the experimental lower bound.

Having determined the p.d.f's for the four $\delta$ 's, we now turn to the evaluation of $\mathcal{S}$ as defined at the beginning of this section. We use the approach defined in ref. ${ }^{19}$ to evaluate the relevant hadronic matrix elements, warning the reader about the large uncontrolled theoretical uncertainties that affect this evaluation. Let us focus for concreteness on the
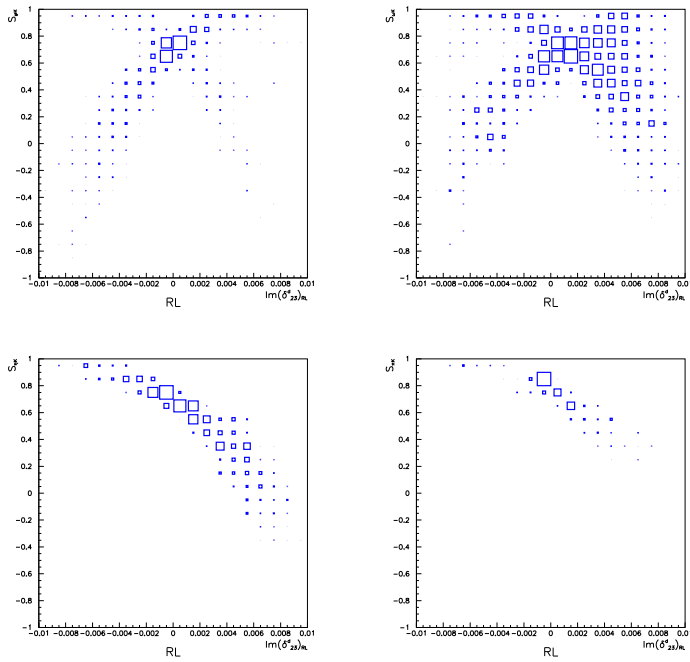

Figure 10. From top to bottom and from left to right, p.d.f.'s for $\mathcal{S}$ for $B$ decays to $\phi K_{S}, \omega K_{S}, \eta^{\prime} K_{S}$ and $\pi K_{S}$ as a function of $\operatorname{Im}\left(\delta_{23}^{d}\right)_{\mathrm{RL}}$.

effects of $\left(\delta_{23}^{d}\right)_{\mathrm{RL}}$. Imposing that the $B R$ 's are correctly reproduced, we obtain the estimates of $\mathcal{S}$ for the $\phi K_{s}, \eta^{\prime} K_{s}, \omega K_{s}$ and $\pi^{0} K_{s}$ final states reported in Fig. 10. One can see that $\left(\delta_{23}^{d}\right)_{\mathrm{RL}}$ insertions can produce sizable deviations from the SM expectations for $\mathcal{S}$ in the $\eta^{\prime} K_{s}$ and $\omega K_{s}$ channels. Similar results hold for the other $\delta$ 's.

Another place where $\delta_{23}^{d}$ mass insertions can produce large deviations from the SM is $\Delta m_{s}$. In this case, hadronic uncertainties are under control, thanks to the Lattice QCD computation of the relevant matrix elements, ${ }^{20}$ and the whole computation is at the same level of accuracy as the SM one. $^{21}$ Considering for example the contribution of $\left(\delta_{23}^{d}\right)_{\mathrm{RR}}$ mass insertions, starting from the constraints in Fig. 9, one obtains the p.d.f. for $\Delta m_{s}$ reported in Fig. 11, where for comparison we also report the compatibility plot within the SM. ${ }^{1}$ Much larger values are possible in the SUSY case, generally accompanied by large values of the CP asymmetry in $B_{s} \rightarrow J / \psi \phi$ : both would be a clear signal of NP to be revealed at hadron colliders. 

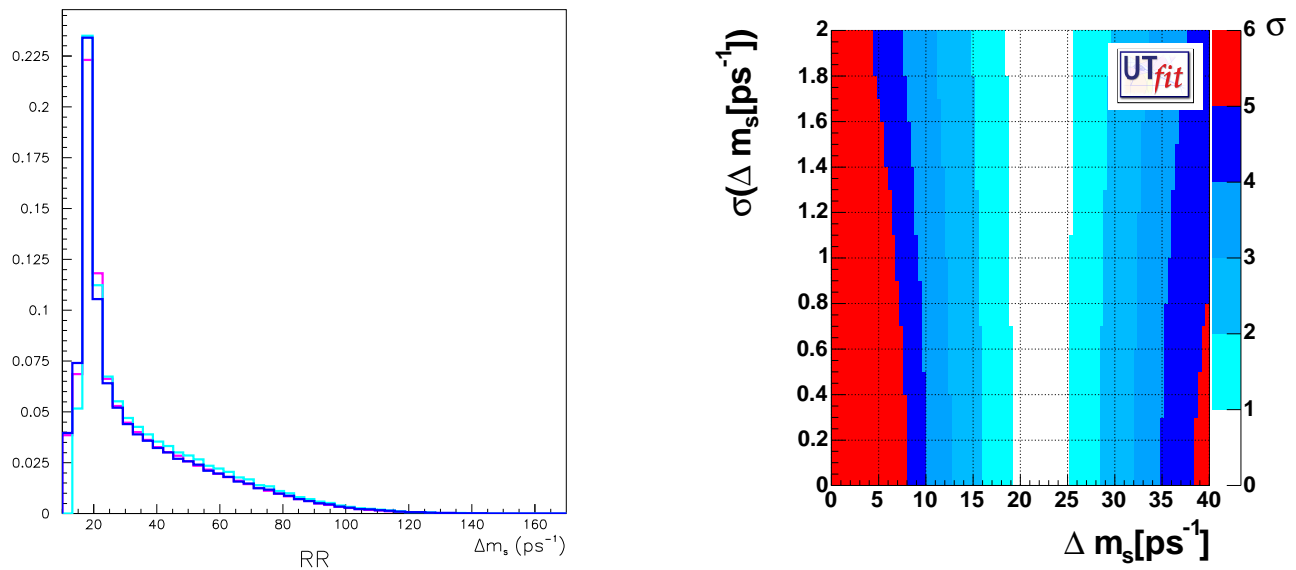

Figure 11. Left: p.d.f. for $\Delta m_{S}$ obtained considering $\left(\delta_{23}^{d}\right)_{\mathrm{RR}}$ and the SUSY parameters given in the text, as determined by $B \rightarrow X_{s} \gamma$ (violet), $B \rightarrow X_{s} l^{+} l^{-}$(light blue) and all constraints (dark blue). Right: compatibility plot for $\Delta m_{s}$ in the SM.
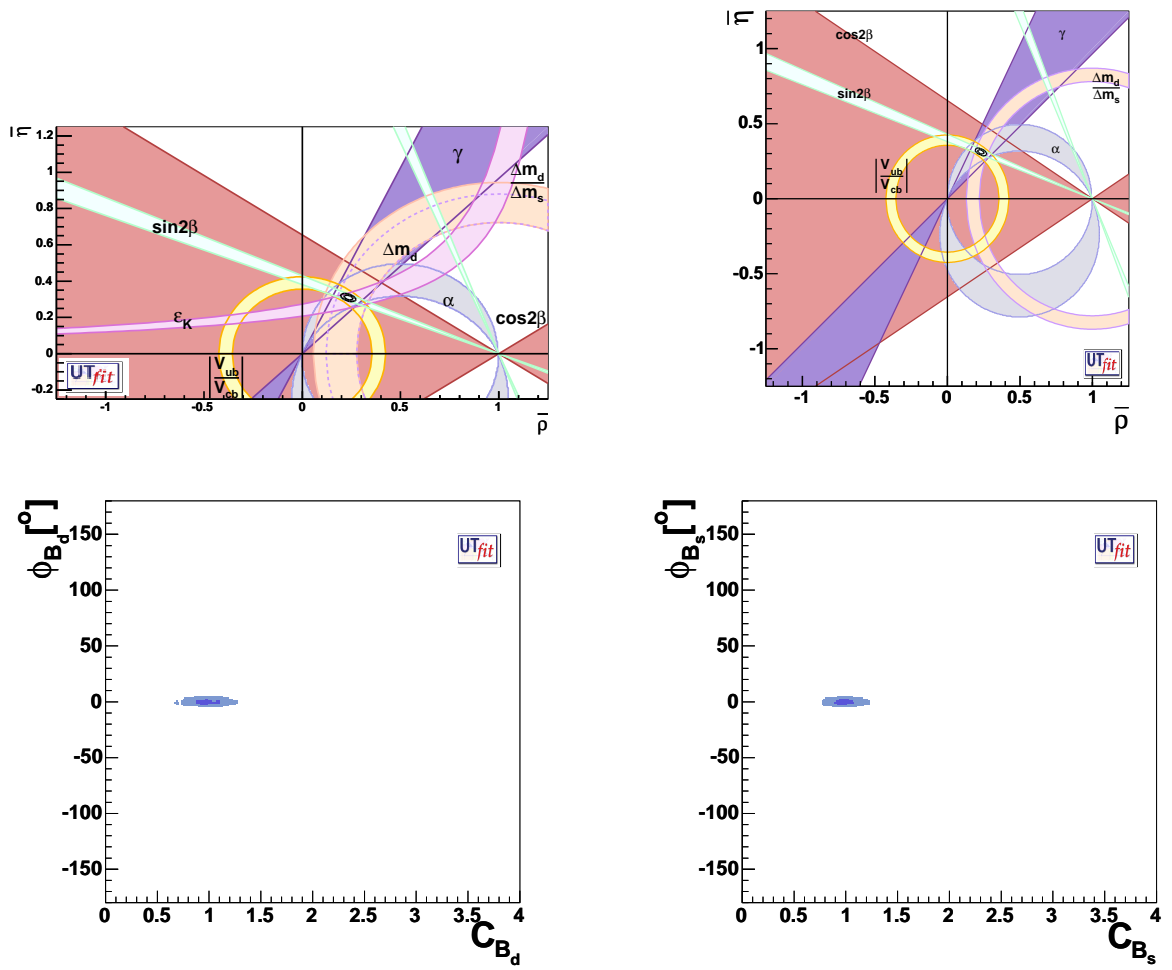

Figure 12. Outlook for Lepton-Photon 2009: the SM UT (top left), the UUT (top right), the $\phi_{B_{d}}$ vs. $C_{B_{d}}$ plane (bottom left) and the $\phi_{B_{s}}$ vs. $C_{B_{s}}$ plane (bottom right). See the text for details. 


\section{Conclusions and Outlook}

Let us summarize the results presented in this talk in four messages:

1 The recent results from $B$ factories make the UT fit overconstrained. This allows us to simultaneously fit SM CKM parameters and NP contributions to $\Delta F=2$ transitions, in the most general scenario with NP also affecting $\Delta F=1$ decays. With present data, the SM-like solution in the first quadrant for the UT is strongly favoured (see Fig. 2). The nonstandard solution in the third quadrant has only $4 \%$ probability.

2 From the generalized UT analysis, we can conclude that NP contributions to $\Delta B=2$ transitions can be of $\mathcal{O}(1)$ if they carry the same weak phase of the SM, otherwise they have to be much smaller or vanishing (see Fig. 4). New sources of flavour and CP violation must therefore be either absent (MFV) or confined to $b \rightarrow s$ transitions. The latter possibility is naturally realized in many NP scenarios.

3 In MFV models, the UUT can be determined, independently of NP contributions, with an accuracy comparable to the SM analysis. Together with the available data on $B \rightarrow X_{s} \gamma, B \rightarrow X_{s} l^{+} l^{-}$and $K^{+} \rightarrow \pi^{+} \nu \bar{\nu}$, this allows to derive stringent upper bounds on other rare $K$ and $B$ decays. Sizable enhancements with respect to the SM are excluded, while strong suppressions are still possible at present.

4 Although the constraints from $B \rightarrow X_{s} \gamma$ and $B \rightarrow X_{s} l^{+} l^{-}$are becoming more and more stringent, NP in $b \rightarrow s$ transitions is still allowed to a large extent and might produce sizable deviations from the SM in the time-dependent $\mathrm{CP}$ asymmetries in $b \rightarrow s$ nonleptonic decays and in $B_{s}-\bar{B}_{s}$ mixing. This situation can be realized in SUSY models, where detailed computations of the deviations from the SM can be performed.

We are bound to witness further improvements in the experimental and theoreti- cal inputs to the above analysis in the near future. In the next few years, the UUT analysis might well become the standard analysis, NP contributions to $\Delta F=2$ transitions will be either revealed or strongly constrained, and rare decays will provide stringent bounds on NP in $\Delta F=1$ processes or, hopefully, show some deviation from the SM expectation. In Fig. 12 I show a pessimistic view of what we might see at Lepton-Photon 2009, in the dull scenario in which everything remains consistent with the SM. ${ }^{4}$ Also in this case, however, flavour physics will remain a crucial source of information on the structure of NP. This information is complementary to the direct signals of NP that we expect to see at the LHC.

I conclude reminding the reader that, for reasons of space, I had to omit several very interesting topics, including in particular lepton flavour violation and electric dipole moments, which might also reveal the presence of NP in the near future.

\section{Acknowledgments}

I am very much in debt to my flavour collaborators: C. Bobeth, M. Bona, A.J. Buras, M. Ciuchini, T. Everth, E. Franco, V. Lubicz, G. Martinelli, A. Masiero, F. Parodi, M. Pierini, P. Roudeau, C. Schiavi, A. Stocchi, V. Vagnoni, S. Vempati, O. Vives and A. Weiler. I acknowledge the support of the EU network "The quest for unification" under the contract MRTN-CT-2004-503369.

\section{References}

1. M. Bona et al. [UTfit Collaboration], JHEP 0507, 028 (2005).

2. J. Charles et al. [CKMfitter Group], Eur. Phys. J. C 41, 1 (2005).

3. J. M. Soares and L. Wolfenstein, Phys. Rev. D 47, 1021 (1993); N. G. Deshpande et al., Phys. Rev. Lett. 77, 4499 (1996); J. P. Silva and L. Wolfenstein, Phys. Rev. D 55, 5331 (1997); A. G. Cohen et al., Phys. Rev. Lett. 78, 2300 (1997); Y. Grossman et al., Phys. 
Lett. B 407, 307 (1997).

4. M. Bona et al. [UTfit Collaboration], arXiv:hep-ph/0509219.

5. S. Laplace et al., Phys. Rev. D 65, 094040 (2002); M. Ciuchini et al., eConf C0304052, WG306 (2003); Z. Ligeti, Int. J. Mod. Phys. A 20, 5105 (2005); F. J. Botella et al., Nucl. Phys. B 725, 155 (2005); K. Agashe et al., arXiv:hep$\mathrm{ph} / 0509117$.

6. G. D'Ambrosio et al., Nucl. Phys. B 645, 155 (2002).

7. A. J. Buras et al., Phys. Lett. B 500, 161 (2001).

8. C. Bobeth et al., Nucl. Phys. B 726, 252 (2005).

9. A. Ali et al., Phys. Rev. D 66, 034002 (2002); G. Hiller and F. Kruger, Phys. Rev. D 69, 074020 (2004); G. Buchalla et al., JHEP 0509, 074 (2005);

10. G. Burdman, Phys. Rev. D 57, 4254 (1998).

11. V. V. Anisimovsky et al. [E949 Collaboration], Phys. Rev. Lett. 93031801 (2004); A. Alavi-Harati et al. [The E799II/KTeV Collaboration], Phys. Rev. D 61 072006 (2000); R. Barate et al. [ALEPH Collaboration], Eur. Phys. J. C 19213 (2001); B. Aubert et al. [BABAR Collaboration], Phys. Rev. Lett. 93091802 (2004); A. Abulencia et al. [CDF Collaboration], arXiv:hep-ex/0508036.

12. A. Masiero et al., Phys. Rev. D 64, 075005 (2001).

13. K. Agashe et al., Nucl. Phys. B 719, 165 (2005); G. Martinelli et al., JHEP 0510, 037 (2005).

14. T. Moroi, Phys. Lett. B 493, 366 (2000); D. Chang et al., Phys. Rev. D 67, 075013 (2003); R. Harnik et al., Phys. Rev. D 69, 094024 (2004).

15. Y. Grossman and M. P. Worah, Phys. Lett. B 395, 241 (1997); M. Ciuchini et al., Phys. Rev. Lett. 79, 978 (1997); D. London and A. Soni, Phys. Lett. B 407, 61 (1997); Y. Grossman et al., Phys. Rev. D 58, 057504 (1998); M. Ciuchini et al., arXiv:hep-ph/0407073.

16. See the session of WG4 on this topic at the CKM 2005 workshop.

17. M. Ciuchini et al., arXiv:hep-ph/0507290.

18. F. Gabbiani et al., Nucl. Phys. B 477, 321 (1996); R. Barbieri and A. Strumia, Nucl. Phys. B 508, 3 (1997); A. L. Kagan and M. Neubert, Phys. Rev. D 58, 094012
(1998); S. A. Abel et al., Phys. Rev. D 58, 073006 (1998); A. Kagan, arXiv:hepph/9806266; R. Fleischer and T. Mannel, Phys. Lett. B 511, 240 (2001); T. Besmer et al., Nucl. Phys. B 609, 359 (2001); E. Lunghi and D. Wyler, Phys. Lett. B 521, 320 (2001); M. B. Causse, arXiv:hepph/0207070; G. Hiller, Phys. Rev. D 66, 071502 (2002); S. Khalil and E. Kou, Phys. Rev. D 67, 055009 (2003); Phys. Rev. Lett. 91, 241602 (2003); eConf C0304052, WG305 (2003); Phys. Rev. D 71, 114016 (2005); G. L. Kane et al., Phys. Rev. D 70, 035015 (2004); S. Baek, Phys. Rev. D 67, 096004 (2003); K. Agashe and C. D. Carone, Phys. Rev. D 68, 035017 (2003); J. F. Cheng et al., Phys. Lett. B 585, 287 (2004); D. Chakraverty et al., Phys. Rev. D 68, 095004 (2003); J. F. Cheng et al., Nucl. Phys. B 701, 54 (2004); E. Gabrielli et al., Nucl. Phys. B 710, 139 (2005); S. Khalil, Mod. Phys. Lett. A 19, 2745 (2004); Phys. Rev. D 72, 035007 (2005).

19. M. Ciuchini et al., Phys. Rev. D 67, 075016 (2003) [Erratum-ibid. D 68, 079901 (2003)].

20. D. Becirevic et al., JHEP 0204, 025 (2002).

21. M. Ciuchini et al., Nucl. Phys. B 523, 501 (1998); A. J. Buras et al., Nucl. Phys. B 586, 397 (2000). 